\title{
Missing Item Prediction and its Recommendation Based on Users
}

\section{Approach in E-Commerce}

\author{
Mr. Himanshu M. Deulkar \\ ME (CSE), Shree Hanuman Vyayam Prasarak Mandal \\ College of Engineering \& Technology, Amravati
}

\author{
Prof. R. R. Shelke \\ Astt. Professor, Shree Hanuman Vyayam Prasarak \\ Mandal College of Engineering \& Tech, Amravati
}

\begin{abstract}
The Internet is one of the fastest growing areas of information gathering. Due to the tremendous amount of data on internet, web data mining has become very necessary. Predicting the missing items form dataset is indefinite area of research in Web Data Mining. Current approaches use association rule mining techniques which are applied to fixed amount of items. Numbers of techniques were used for generating items but not a single technique was used to take its advantage. Now in order to minimize the cost of mining for huge database and to provide online prediction, paper proposes effective method for prediction. This has reduced complex algorithm that were used to generate items and strongly worked on dissimilar approach that is proposed.
\end{abstract}

KEYWORD : Missing Item prediction on online shopping portal, Item Recommendation in ecommerce website, Paper on Prediction and recommendation of item

\section{INTRODUCTION}

Internet is a global system of inter connected network and has gained huge amount of popularity in recent time. This accumulated various businesses to come online and make easier for them to reach users of different part of the globe. Also business like ecommerce i.e. online Shopping portal became more convenient for people as they were able to purchase a product on one click. Also This Business has provided different range of products for online users.

In this work, a next logical step is taken that allow any item to be treated as a class and its value is predicted depending upon on the presence or absence of other items. Product will predicted depending upon his past history their dislike. For example There are 3 users Louis, Christian and Ji Moung. All three of them have dislike one product i.e "X-Laptop" now depending upon it they will be considered to be in one group. Now they Scenario will be if Ji Moung purchases any product it will be predicted for other two i.e for Louis and Christian.

The approaches where it look for "similar friends" or "similar product items" directly, It will first look for the target user's dissimilar "enemy" (i.e., antonym of "friend"), and furthermore, It will look for the "possible friends" of E-commerce target user, according to "enemy's enemy is a friend" afterwards, the product items preferred by the target user's "possible friends" are regarded as the recommendation candidates for target user; likewise, for the product items preferred by target user, It first determine their "possibly similar product items" based on "enemy's enemy is a friend" rule of Structural Balance Theory, and regard them as the recommendation candidates for target user.

The Ecommerce recommendation problem is formalized and afterwards, the proposed work motivation is demonstrated. A recommendation approach over big rating data in E-commerce, is put forward. A set of experiments are designed and deployed in later section for validating feasibility in terms of recommendation accuracy, recall and efficiency. In Last Section, I summarize the whole proposed work and suggest the possible research directions in the future.

\section{LITERATURE REVIEW \& RELATED WORK}

Association rule mining find all the rule that give satisfaction of getting minimum support and minimum confidence condition. Most works allow the user to specify a QoS-based service selection policy:[4] its aim is to give result with respect to the queries fired or used from other users.[7] its objective it to get most favourable result [3] and [6] recommends use of various parameter without generating any common key.

The goal was to create a specific class using association rule. This will be use to reduce the complexity. 


\section{www.ijtsrd.com}

Almost classification rule is used on data in a table format, where the attribute are already defined. In our algorithm we do not have any predefined set of values. Here the users past history is important depending on which prediction is delivered. The Algorithm works efficiently and reduces the complexity within the association of data.

In time-aware recommendation is introduced, where time is considered as an important factor for predicting product quality. However, work only discusses the objective quality prediction, without considering the subjective preferences of different users. Matrix factorization technique is introduced in [8] to realize the recommendation purpose; however, if the user-product rating matrix is very sparse, the recommendation effect is not as good as expected (e.g., overfitting problem). In [6], a Monte Carlo algorithm named MCCP is brought forth to measure different users' personalized preferences towards different product items. According to MCCP, user target's similar friends can be found by trust propagation; and afterwards, the missing product item quality could be predicted based on the obtained similar friends. Generally, MCCP can work very well if user target has similar friends. However, as introduced previously in this proposed work, I only focus on the specific recommendation situations when user target does not have similar friends; therefore, prediction accuracy and recall of MCCP are not as good as expected, which has been validated by the experiments. In our previous work, a recommendation approach USER BASED PARAMETER is put forward, for dealing with the specific recommendation scenarios where user target has no similar friends and the product items liked by user target do not have similar product items. While user based parameter approach has two shortcomings. First, only "enemy's enemy is a friend" rule is recruited in user based parameter. Second, user based parameter only adopts user-based CF recommendation, while neglects item-based $\mathrm{CF}$ recommendation as well as their integration. Therefore, the recommendation effect of user based parameter is not as satisfactory as expected. In view of the shortcomings of above approaches, I put forward a novel product item recommendation approach User based parameter. Through "enemy's enemy is a friend" and "enemy's friend is an enemy" rules in Structural Balance Theory, user based parameter can make full use of the valuable structural balance information hidden in user-product purchase network, and further make precise product item recommendation. Moreover, user based parameter integrates both user-based $\mathrm{CF}$ recommendation and item-based $\mathrm{CF}$ recommendation; therefore, the recommendation recall could be improved. Finally, through a set of experiments deployed on MovieLens$1 \mathrm{M}$ dataset, I validate the feasibility of User based parameter in terms of recommendation accuracy, recall and efficiency.

\section{EXISTING SYSTEM}

Present algorithm in this spectrum use fast and effective technique. This uses association rule mining techniques. First approach uses set of item to construct association rule between items. Also if there is no certainty in the occurrence then it will be measured but bayesian technique. This method produces high support and high confidence rules. This technique proves to appear better than the traditional techniques in association rule mining. But the cons of this technique are complexity increases with the increase in average length of items. Yet another method to predict missing items uses Boolean vector and the relational AND operation to discover frequent item sets [3] [6] without generating candidate items directly generate the association rules. Association rules are used to recognize the correlation among a set of items in database.

Boolean values (either 0's or 1's). The frequent item sets are generated from the Boolean matrix. The association rules generated from the frequent item set for prediction. The next item set i.e the content of incoming shopping cart will also be represented by a Boolean vector and "AND" operation is performed with each transaction vector to generate the association rules. Lastly the rules are combined by Dempster's rule of combination to get the predictions. The advantages of this technique are that it doesn't generate candidate item sets, it uses only a single pass over the database, the memory consumption is low and the processing speed is more as compared to the previous technique. The disadvantage of this approach is that the use of a Boolean matrix cannot handle huge amount of data. This disadvantage restricts the use of this technique in online applications where huge amount of data is generated. The major drawback of the above mentioned technique is the rule generation 
complexity. Generating rules from a huge amount of data involves a lot of high memory and time complexity. In DS - ARM technique the rule generation complexity increases by huge amount as the average transaction length increases and in the technique using fast algorithms though the rule generation complexity is lower the data structures used are not capable of handling huge amounts of data. In addition to these techniques another proposed work discusses a graph based approach towards association rule mining. This proposed work suggests an algorithm called Combo Matrix algorithm which predicts missing items using associative classification mining. The data structure used to store a graph is an adjacency matrix with a slight modification wherein the diagonal elements contain the list of vertices the adjacent to the given vertex. The advantages of this approach are reduced rule generation complexity however; the data structure used and the algorithm proposed do not perform well for large data sets since an adjacency matrix is used thereby having space complexity of $\mathrm{O}|\mathrm{V} 2|$ where $\mathrm{V}$ is the number of vertices and equal to the number of items present in the database. The method proposed in this proposed work aims at reducing the rule generation complexity by classifying the items and then constructing a graph from it and using the graph for prediction purpose.

\section{ANALYSIS OF PROBLEMS}

Two things that complicate the path that is number of item to be predicted as there are limitation. Here an item which is associated with most of the user can be recommended and same with the prediction. Secondly the association of user and predicting item for them was bit complicated as whose item should be predicted to whom was merely a complicated situation where algorithm suggested that all the product should be predicted if user grouped accordingly. Classical association mining usually ignores this aspect, perhaps because negated items tend to increase significantly the total number of rules to be considered; another reason can be that rules with mutually contradicting consequents are not so easy to combine.

\section{PROPOSED WORK AND OBJECTIVE}

1. First module is of item recommendation where same category products are recommended.
2. Second module is of item prediction where depending upon users past history product will be predicted for the users.

3. It assures quality service for user.

4. It tracks all users activity of review and purchase that creates better user experience.

\section{RESULT}

Initially tests were taken by creating few accounts. Firstly 3 accounts were created and all were made to dislike the same product. And if any one of the user will purchase any item that product will be recommended to other 2 users. So to carry out the whole process user to input must be given by user first account creation and secondly his review depending upon modules will work.

\section{REFERENCES}

[1] Lianyong Qi, Xiaolong Xu, Xuyun Zhang, Wanchun Dou, Chunhua Hu, Yuming Zhou, Jiguo Yu

" "Structural Balance Theory-based E-commerce Recommendation over Big Rating Data” 2016 IEEE.

[2] Kasun Wickramaratna, Miroslav Kubat,and Kamal Premaratne,"Predicting Missing Items in Shopping Carts" IEEE TRANSACTIONS ON KNOWLEDGE AND DATA ENGINEERING, VOL. 21, NO. 7, JULY 2009.

[3] Junqiang Liu, Member, Ke Wang, and Benjamin C.M. Fung,"Mining High Utility Patterns in One Phase without Generating Candidates" IEEE TRANSACTIONS ON KNOWLEDGE AND DATA ENGINEERING, VOL. 28, NO. 5, MAY 2016

[4] R. R. Shelke, Dr. R. V. Dharaskar, Dr. V. M. Thakare, "Analysis of User Movement Behaviour Pattern Mining Approaches for Mobile Environments", International Journal of Advanced Research in Computer Science, Volume 5, No. 4, April 2014, ISSN No. 0976-5697.

[5] M. Mayilvaganan,D. Kalpanadevi"Comparison of Classification Techniques for predicting the Cognitive Skill of Students in Education Environment" 2014 IEEE International Conference on Computational Intelligence and Computing Research

[6] Junqiang Liu,Ke Wang, and Benjamin C.M. Fung"Mining High Utility Patterns in One Phase 
without Generating Candidates" IEEE [7] R. R. Shelke, Dr. R. V. Dharaskar, Dr. V. M. TRANSACTIONS ON KNOWLEDGE AND DATA Thakare, "DATA MINING FOR MOBILE DEVICES ENGINEERING, VOL. 28, NO. 5, MAY 2016.

USING WEB SERVICES", International Conference Innovations in Information Embedded and on Industrial automation And Computing ( ICIAC - Communication Systems ICIIECS 15 12th \& 13th April 2014), Jhulelal Institute of Technology, Nagpur

[10] "An Improved Collaborative Filtering Recommendation Algorithm not Based on Item

[8] Jos'e Cazal "Predictive models of economic systems based on data mining" International Workshop on Data Mining with Industrial Applications 2015

[9] "An Approach for Predicting the Missing Itemsfrom Large Transaction Database" IEEE Sponsored 2nd International Conference on CognltlVelnlormatlcs \& Cognitive Computing IICCrCC'151

[11] "Predicting Missing Items in a Shopping Cart using Apriori Algorithm" International Journal of Engineering Trends and Technology (IJETT) Volume 20 Number 4 - Feb 2015 